\title{
Un diagnóstico de infraestructura geodésica en la región de Centroamérica y el Caribe
}

\author{
David Avalos Naranjo* \\ Carlos E. Figueroa ${ }^{+}$ \\ Wilmer Medrano Silva ${ }^{\triangle}$ \\ Christopher Ballesteros \\ Vinicio Robles Pereira ${ }^{\circ}$ \\ Alvaro Alvarez Calderón $\bullet$ \\ Leopoldo Tavera Polanco ${ }^{\wedge}$ \\ Oscar Meza
}

\begin{abstract} of users like surveyors and high precision cartographers.

* Instituto Nacional de Estadística y Geografía, México.

+ Centro Nacional de Registros, El Salvador.

$\triangle \quad$ Instituto Nacional de Estudios del Territorio, Nicaragua.

- Instituto Geográfico Nacional "Tommy Guardia", Panamá.

- Instituto Geográfico Nacional, Guatemala.

- Instituto Geográfico Nacional, Costa Rica.

- Instituto Cartográfico Militar, República Dominicana.

- Instituto de la Propiedad, Honduras.
\end{abstract}

The document shows a summary of the existing geodetic infrastructure in 8 countries of Central America and the Caribbean. Through maps and tables it is offered updated concrete figures about the amount and location of the control networks in horizontal, vertical and gravimetric fields. The federal agencies in charge of establishing the national official reference frame explain the technical characteristics of their infrastructure at the time of describing their vision to progress for satisfaction

By analyzing the contents it has been identified a set of similarities and discrepancies in the degree of development of the infrastructure among countries. Currently the regional geodetic infrastructure has achieved an impulse enough to satisfy minimal operation requirements. As a coordinated group, the 8 agencies gather a significant set of control marks on the terrain and data bases in constant 
improvement. From a perspective based in world-wide trends, it can be said that the infrastructure needed for future requirements is compromised due to symptomatic budget insufficiency, which in cases has driven to stop the progress in vertical and gravimetric branches. It is concluded making a recommendation to encourage the specialized training, from where the personnel in the geographic agencies can design custom projects of local extent, able to balance the progress in the three branches of geodetic control.

Key words: geodesy, Central America, infrastructure, international cooperation.

\section{Resumen}

El documento presenta un sumario de la infraestructura geodésica existente en ocho países de la región Centroamericana y del Caribe. A través de mapas y tablas se ofrecen cifras actualizadas concretas sobre cantidad y ubicación de las redes de control horizontal, vertical y gravimétrico. Las agencias federales encargadas de establecer el marco de referencia oficial nacional explican características técnicas de su infraestructura y describen su visión de avance para satisfacer a usuarios como topógrafos y generadores de cartografía de alta precisión.

Analizando los contenidos se identificaron similitudes y discrepancias en el grado de avance entre países. Hasta el momento la infraestructura geodésica regional ha logrado ser impulsada para satisfacer requerimientos mínimos de operación. Las agencias ostentan un conjunto significativo de marcas de referencia sobre el terreno y bases de datos en actualización constante. Visto esto desde una perspectiva de avance y tendencias mundiales, puede decirse que el cubrimiento de necesidades futuras está comprometido debido a insuficiencias presupuestales sintomáticas que en casos han llevado a detener el avance en el control vertical y gravimétrico. Se concluye haciendo una recomendación a incentivar la capacitación especializada, desde la cual el personal de las agencias geográficas podrá producir proyectos locales hechos a la medida para equilibrar los avances en las tres vertientes del control geodésico.

Palabras clave: geodesia, Centroamérica, infraestructura, colaboración internacional.

\section{Introducción}

Tablas de datos, mapas de ubicación, y textos de análisis son los elementos sustantivos de este diagnóstico de infraestructura geodésica. Con esto se ofrece al lector un apoyo para comprender los avances y planes de desarrollo en 8 países de la región Centroamericana y del Caribe.

La infraestructura geodésica, vista desde una administración federal, es el conjunto de datos y marcas físicas establecidas sobre un territorio nacional que aportan la georeferencia primaria desde la cual toda la información geográfica adquiere atributos de posición consistente. La característica de consistencia per- 
mite evitar ambigüedades, disminuyendo la posibilidad de encontrar conflictos al combinar capas de información territorial que proviene de distintas fuentes. Esta infraestructura resulta indispensable en la construcción de mosaicos de modelos tanto de temas topográficos como referentes al medio ambiente y sociales de fines estadísticos.

A nivel nacional cada administración conviene en la adopción de estándares que funcionan al interior del país pero comúnmente son distintos de los adoptados en naciones colindantes. Esta falta de empate llega a impedir la compatibilidad de datos que es crítica en trabajos de interés binacional o regional. De aquí la relevancia de mantener abierta la comunicación interinstitucional impulsando el intercambio de información geodésica y la renovación tecnológica.

Con esta visión se inició el proyecto de cooperación técnica del IPGH llamado Monitoreo del geoide para México, Centroamérica y el Caribe, que consiste en la apertura de oportunidades de intercambio tecnológico orientadas a construir acuerdos de compatibilidad en infraestructura geodésica (Avalos, 2011). Aquí se presenta en gran parte los primeros resultados de este proyecto, organizando una considerable cantidad de información sobre el estado que guarda actualmente la infraestructura geodésica de ocho agencias geográficas nacionales de la región. Las representaciones nacionales que participaron corresponden al Instituto Nacional de Estadística y Geografía (INEGI) de México, Instituto Geográfico Nacional (IGN) de Guatemala, Centro Nacional de Registros (CNR) de El Salvador, Instituto de la Propiedad (IP) de Honduras, Instituto Nacional de Estudios del Territorio (INETER) de Nicaragua, Instituto Geográfico Nacional (IGNCR) de Costa Rica, Instituto Geográfico Nacional "Tommy Guardia" (IGNTG) de Panamá y al Instituto Cartográfico Militar (ICM) de República Dominicana. Estos institutos han sido representados por los ocho autores. De manera consistente a lo largo del documento se hará referencia a las ocho instituciones como las agencias.

Los contenidos se estructuran en tres secciones principales en las que se informa y discute la actual infraestructura geodésica por sus vertientes de control vertical, gravimétrico y horizontal. En cada sección se añade información aclaratoria sobre la orientación de programas de trabajo junto con una breve descripción del desarrollo esperado en el corto plazo.

Con esta aportación, las propias administraciones federales de la región encuentran ya datos útiles para crear un panorama con perspectiva imparcial sobre el grado de avance relativo. Adicionalmente se aportan elementos básicos de la tendencia de avance global que sirven de sustento a la obtención de conclusiones menos relativas. De esta manera se llega a mostrar que existe un desbalance en el apoyo al desarrollo de las tres vertientes de infraestructura geodésica. La vertiente horizontal es comúnmente priorizada ante limitantes presupuestales que en ocasiones llegan incluso a inhibir los avances en las vertientes gravimétrica y vertical. 
Estas piezas de información pueden apoyar la toma de decisiones al facilitar la identificación de temas y zonas prioritarias de desarrollo regional. Solamente al procurar un balance entre las tres vertientes se abre la puerta a la implementación de aplicaciones modernas entre las que se encuentran técnicas geofísicas de prospección y el desarrollo de modelos geoidales precisos; éste último indispensable para el posicionamiento vertical por tecnología GNSS.

\section{Infraestructura de referencia vertical}

Desde mediados del siglo Xx el sistema de alturas, junto con la técnica de medición de elevaciones que se aplican para establecer la referencia vertical de datos geográficos han sido elementos homogéneos en la región. Sin embargo, al hablar de los criterios de operación y de la visión a futuro sobre el control geodésico vertical, existen diferencias que a continuación se documentan.

Cada agencia hace corresponder sus redes de elevación a un punto de origen o dátum independiente. La Tabla 1 presenta el nombre del dátum que actualmente se aplica como punto de origen oficial (véanse las "Tablas de resumen sobre infraestructura geodésica"). Estos hacen referencia al nivel medio del mar registrados en épocas y mareógrafos específicos entre los cuales, hasta donde nuestro conocimiento alcanza, no se ha logrado generar algún estudio sistemático de discrepancias entre naciones.

Los valores de altura $(\mathrm{H})$ asociados a cada monumento geodésico de estas redes provienen de un cálculo de acumulación de desniveles $(\Delta \mathrm{L})$. La ecuación de propagación de alturas utilizada se lee: la altura ortométrica de una marca numerada $n$ es igual a la altura $H_{1}$ de la primera marca situada junto al mareógrafo que dictó el nivel medio del mar, más la suma de diferencias de altura $\Delta L_{i}$ medidas entre marcas consecutivas desde la primera hasta llegar a la marca $n$. Es decir:

$$
H_{n}=H_{1}+\sum_{i-1}^{n} \Delta L_{i}
$$

La Figura 1 muestra la distribución espacial aproximada de sitios registrados con referencia vertical disponible en cada agencia nacional. Esta información gráfica se complementa con datos de la Tabla 1, donde en forma sintetizada se describe un panorama de los recursos tecnológicos y humanos con los que actualmente se mantiene el control geodésico vertical. Debe considerarse que las cifras plasmadas sobre cantidad de marcas en la columna de 1er., 2do. y 3er. orden son aproximaciones que pueden variar en relación a su constante actualización, la cual es motivada tanto por adhesión de nuevos bancos de nivel establecidos como por la detección de marcas desaparecidas. Algunos programas de trabajo de inventariado en campo permiten producir dicha actualización. 


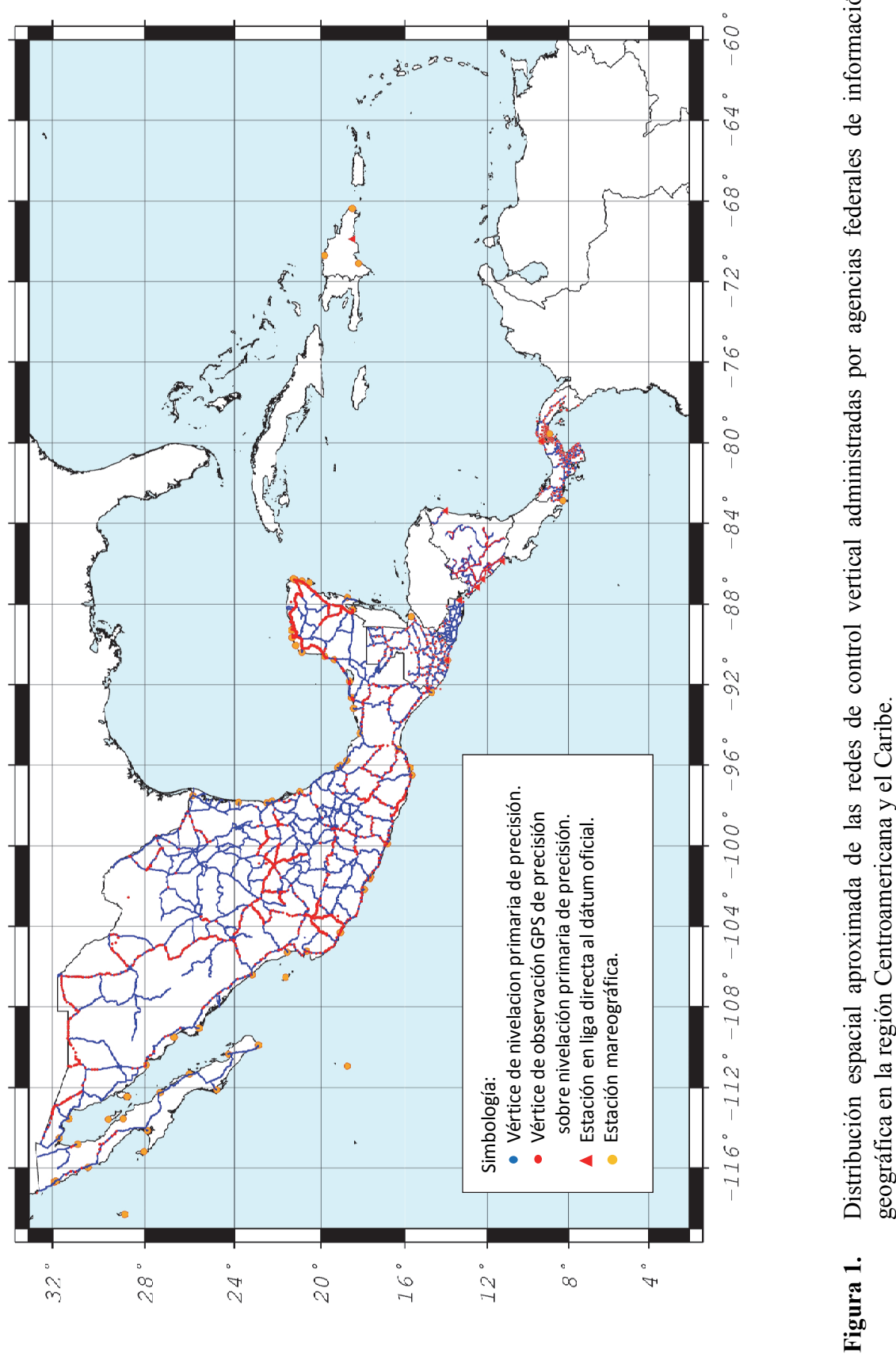


Puede notarse en la Tabla 1 que los mareógrafos locales son considerados como parte de la infraestructura geodésica en las agencias, a pesar de que se encuentran administrados por distintos organismos. Actualmente una baja cantidad de mareógrafos en la región son mantenidos en programas de operación continua, indispensables para monitorear el nivel del mar en relación con el dátum vertical. Diversos estudios (e.g. IPCC, 2007; Church y White, 2006; Peltier, 2001) han mostrado que el nivel medio del mar es una referencia que experimenta cambios de magnitud variable en cualquier costa. De estos estudios se concluye que existe una tendencia promedio de aumento global cercana a los 2 milímetros por año. Debe considerarse que el establecimiento de redes de control vertical sistematizado a nivel nacional se inició de manera paralela por las agencias entre las décadas de los años cuarenta y sesenta. De forma que tras una acumulación de 50 años sobre la fecha de establecimiento de un nivel medio del mar es de esperarse que el cambio de nivel pueda ser medido incluso en decímetros. Esto es significativo si se compara con rangos de error tolerable establecidos por metodología en las propias agencias que forman parte de este estudio.

\section{Acoplamiento con la tecnología GNSS}

Actualmente la técnica de nivelación geodésica continúa considerándose como la herramienta indispensable en mantenimiento y propagación de estas redes; sin embargo es aceptado que alternativamente aplicaciones como la geo-referencia catastral derive sus registros de elevación a partir de tecnología de sistemas satelitales de navegación global (GNSS). En todas las agencias el método utilizado consta de la aplicación de un modelo de alturas geoidales $(\mathrm{N})$ para transformar en alturas ortométricas $(\mathrm{H})$ los datos de altura geodésica $(\mathrm{h})$ obtenidos por GNSS, como se describe en la ecuación siguiente.

$$
\mathrm{H}=\mathrm{h}-\mathrm{N}
$$

El nombre del modelo geoidal recomendado de forma independiente por cada agencia se especifica en la Tabla 1.

\section{Información específica por agencia}

En esta sección presentamos información aclaratoria que permite al lector comprender el estado actual de programas de trabajo y proyectos que inciden sobre las redes de referencia vertical. 


\section{Panamá}

El IGNTG mantiene actividades de actualización y normalización de la base de datos de control geodésico vertical. En los próximos años se espera que las operaciones de nivelación continúen efectuando recuperación de líneas antiguas y densificando la red de control vertical. Los mareógrafos que se utilizaron para generar el Dátum Cristóbal no existen en la actualidad; sin embargo la Autoridad del Canal de Panamá (ACP), mantiene en funcionamiento dos mareógrafos que podrían ser utilizados para estimar cambios regionales en el nivel del mar. Ya se han realizado estudios para efecto de ubicar nuevas estaciones mareográficas que permitan determinar la relación entre dátum altimétrico, dátum hidrográfico y línea de costa en diversas zonas litorales. Esto apoyará los trabajos futuros de liga con marcos verticales de países vecinos.

\section{Nicaragua}

Actualmente en el INETER se revisan y modernizan los registros de control geodésico vertical para incrementar la oportunidad de respuesta en atención de usuarios. Con respecto al dátum Corinto, éste es una realización compuesta del nivel medio del mar observado en tres mareógrafos: Corinto, San Juan del Sur y Puerto Sandino. El NMM de los registros hechos entre las décadas de los cincuenta y sesenta fue tomado como restricción en los tres puntos para generar los valores de altura desde el océano Pacífico. En la costa del Atlántico se designó al mareógrafo de Puerto Cabezas como referencia para una red independiente y de menor extensión que da servicio a la región noreste de Nicaragua.

\section{México}

El dátum NAVD88 está asociado a un sólo mareógrafo en Rimousky, Canadá. Las alturas propagadas en México desde ese dátum contienen correcciones gravimétricas calculadas con la aproximación de Helmert. Actualmente en el INEGI se avanza en la determinación de alturas asociadas a ese dátum para el 100\% de marcas de la red geodésica vertical de primer orden. En paralelo se produce y mejora el modelo nacional de alturas geoidales para dar soporte a usuarios de GNSS.

\section{El Salvador}

El CNR desde el año 2004 mantiene un programa constante de actualización, mantenimiento y densificación de la red de nivelación de Primero y Segundo Orden de todo el país, basado en el Datum de La Unión de 1960. Se mantiene vinculada la red a dos mareógrafos establecidos en los principales puertos del país. Recientemente se han reemplazado los equipos de nivelación analógicos por niveles geodésicos electrónicos de alta precisión y se ha iniciado la recuperación de la red de 
puntos de nivelación que contaban con datos de gravimetría, así como la red de Tercer Orden.

\section{Sobre la tendencia regional y global}

Es un hecho que en redes de nivelación de corta y mediana extensión, como son áreas metropolitanas o municipales, la precisión relativa alcanzable es insuperable para otras técnicas de posicionamiento. Por lo anterior, nivelación continúa siendo la herramienta predilecta en aplicaciones como control geodésico y monitoreo de movimientos verticales del terreno. No obstante, la alternativa de posicionamiento vertical mediante GNSS ha logrado, por su bajo costo, absorber una gama de aplicaciones cada vez más amplia entre las que se enlista la producción de modelos digitales del terreno, georeferencia para objetos y registros catastrales, así como el apoyo al posicionamiento de imágenes de percepción remota (e.g. Gibbons Media y Research LLC, 2013).

En años recientes los algoritmos de procesamiento de señales GNSS mejoraron el nivel de precisión de posicionamiento (Hirt et al., 2011), al grado de implementar con éxito metodologías de posicionamiento GPS en el establecimiento de desniveles útiles en ciertos casos de transporte de fluidos por ductos. El bajo costo de esta técnica, aunado al reciente aumento de exactitud en los modelos de altura geoidal ha hecho viable incluso la alternativa de conceptuar a los modelos geoidales como dátum de alturas oficiales. Ejemplos de esto se encuentran en Nueva Zelanda y Canadá (e.g. Amos, 2010; Huang). Agencias geodésicas de otros países en el mundo fortalecen sus programas para incrementar la exactitud de sus modelos geoidales rumbo a la adopción de esa superficie como dátum de alturas y como apoyo en nuevas aplicaciones como el modelado de inundaciones.

\section{Discusión}

Haciendo un análisis exploratorio de la información de la Tabla 1, puede verse que el promedio de territorio asignado a cubrir por cada brigada de nivelación es cercano a $56,000 \mathrm{~km}^{2}$. De este promedio sobresalen los casos Guatemala y Honduras, con más de $100,000 \mathrm{~km}^{2}$ a cubrir por brigada y por el contrario, El Salvador con cerca de $7,000 \mathrm{~km}^{2}$ por brigada, cifra que lo convierte en el país relativamente mejor habilitado de la región.

La información anterior se complementa con las columnas de proyectos y presupuesto de la Tabla 1, que dejan ver como en las agencias de Guatemala, Honduras, Nicaragua, Costa Rica y Panamá se ha llegado a detener la actividad de densificación de la red de control vertical nacional. No obstante, de forma unánime entre las agencias se reconoce que la reactivación de esta actividad coadyuvaría significativamente al impulso de nuevas tecnologías en la generación de información topográfica de calidad homogénea en cada territorio nacional. Luego de una 
discusión entre los autores, desde nuestra perspectiva el principal problema detectado que dificulta desarrollar la actividad, es la limitación en financiamiento que impide adquirir equipo como equialtímetros y miras geodésicos acordes con la actualidad tecnológica. Otro problema relevante como la falta de oportunidades para actualizar la capacitación en metodologías de levantamiento y tratamiento de los datos puede mitigarse al tomar ventaja de las relaciones interinstitucionales existentes.

En cuanto a la definición del dátum oficial es importante resaltar que la adopción de un nivel medio del mar ha permitido a cada agencia ofrecer una referencia consistente al interior de su país. Este nivel de referencia se ha mantenido inmóvil desde su creación hace 50 años o más mientras la red de nivelación continúa incrementando su cobertura o densidad. No obstante es reconocido que la elevada actividad sísmica asociada con movimientos del terreno es característica en la región, lo que genera incertidumbre sobre la actual correspondencia de las alturas con el nivel del mar y el efecto de tales movimientos en la distorsión de las redes con el paso del tiempo. Adicionalmente, el efecto del desnivel entre los océanos del Pacífico y el Caribe aún no ha sido evaluado como factor incidente de distorsión en las redes de nivelación que fueron restringidas al nivel medio de ambos lados del continente.

La diversidad de dátum verticales en la región produce una esperada discrepancia en los valores de elevación para marcas geodésicas comunes entre países vecinos. Causas comunes de estas diferencias son: a) la diferencia en las épocas de datos tomados para determinar el nivel medio del mar; b) la diferencia no medida entre la superficie topográfica marina, que es la suma de efectos por elementos ambientales como: temperaturas promedio, dirección de viento predominante, presión atmosférica, corriente marina y descarga de ríos entre muchos otros (e.g. Foreman et al., 2008; Pagiatakis, 2009); y c) distorsiones independientes que se derivan de errores sistemáticos en desniveles de la red como la posible falta de correcciones ortométricas o correcciones por refracción residual entre otras. La determinación de estas cantidades queda fuera del propósito en este documento; sin embargo se enumeran como puntos que se recomienda abordar en futuros trabajos de confrontación de sistemas de alturas entre países vecinos.

Alternativas consideradas en años recientes para habilitar la compatibilidad de datos de altura a nivel interinstitucional son: la adopción de un dátum regional basado en superficies gravimétricas como el geoide o el cuasi geoide, y la adopción de un sistema de alturas dinámicas realizado exclusivamente por mediciones gravimétricas sobre una red continental de bancos de nivel. Estas opciones son compatibles y pueden ser coincidentes mediante la determinación de un valor de potencial gravimétrico estándar (Wo). 
Por lo anterior, las agencias de la región de Centroamérica y el Caribe consideran ya estas tendencias para apoyar esfuerzos dirigidos a la determinación de una referencia vertical común. Además de haber identificado el impulso que se puede dar en la infraestructura geodésica y el desarrollo nacional asociado, se reconoce la utilidad de las referencias gravimétricas como apoyo en áreas de geofísica y modelado de inundaciones.

\section{Infraestructura de referencia gravimétrica}

Al tiempo de edición de este reporte, las agencias de la región manejan políticas heterogéneas con respecto a la prioridad de generar datos de gravimetría. Esto depende primordialmente del uso o aplicación en programas de trabajo gubernamentales que marcan como requisito la disponibilidad de esta fuente. Aquí se reporta que una casi generalizada carencia de gravímetros en resguardo o en propiedad de las agencias ha sido factor para mantener deshabilitada la actividad de monitoreo del campo de gravedad. En la mayor parte de los países en la región existen bases de datos con gravimetría de levantamientos antiguos, mayormente obtenida por agencias y universidades extranjeras. El Inter-American Geodetic Survey de los Estados Unidos generó datos durante las décadas de los sesentas a ochentas con motivo de producir la Red Internacional de Estandarización de la Gravedad de 1971 (IGSN71) y posteriormente para la Red Latinoamericana de Gravedad de 1977 (RELANG77). En países como Guatemala, El Salvador, Honduras y Nicaragua esta información solo ha sido complementada con un conjunto de mediciones con gravímetro absoluto llevadas a cabo por el National Geodetic Survey (NGS) de los Estados Unidos durante 1996 y en 2001.

Con respecto al dátum gravimétrico, las agencias cuentan con bancos de datos en que los levantamientos de gravimetría relativa fueron asociados a referencias como las redes IGSN71, RELANG77, sitios con gravedad absoluta medida y algunos sin referencia documentada. Para facilitar la comprensión de estos datos, se muestra la Tabla 2 con un resumen de actividad reportada por las agencias (véanse las "Tablas de resumen sobre infraestructura geodésica"). Se advierte que las cifras plasmadas en la columna de información antigua y reciente son aproximadas y podrían variar en relación a procesos actuales de depuración de datos o a recuperación de puntos levantados por fuentes externas. Adicionalmente, la Figura 2 muestra la distribución espacial aproximada de los 81,999 sitios registrados.

Adicional a lo anterior han existido levantamientos por cuenta de instituciones educativas que han decidido colaborar con el Buró Gravimétrico Internacional (BGI). Un total de 18,227 puntos gravimétricos en resguardo del BGI correspondientes a los 8 países se agregaron en el mapa de la Figura 2. 


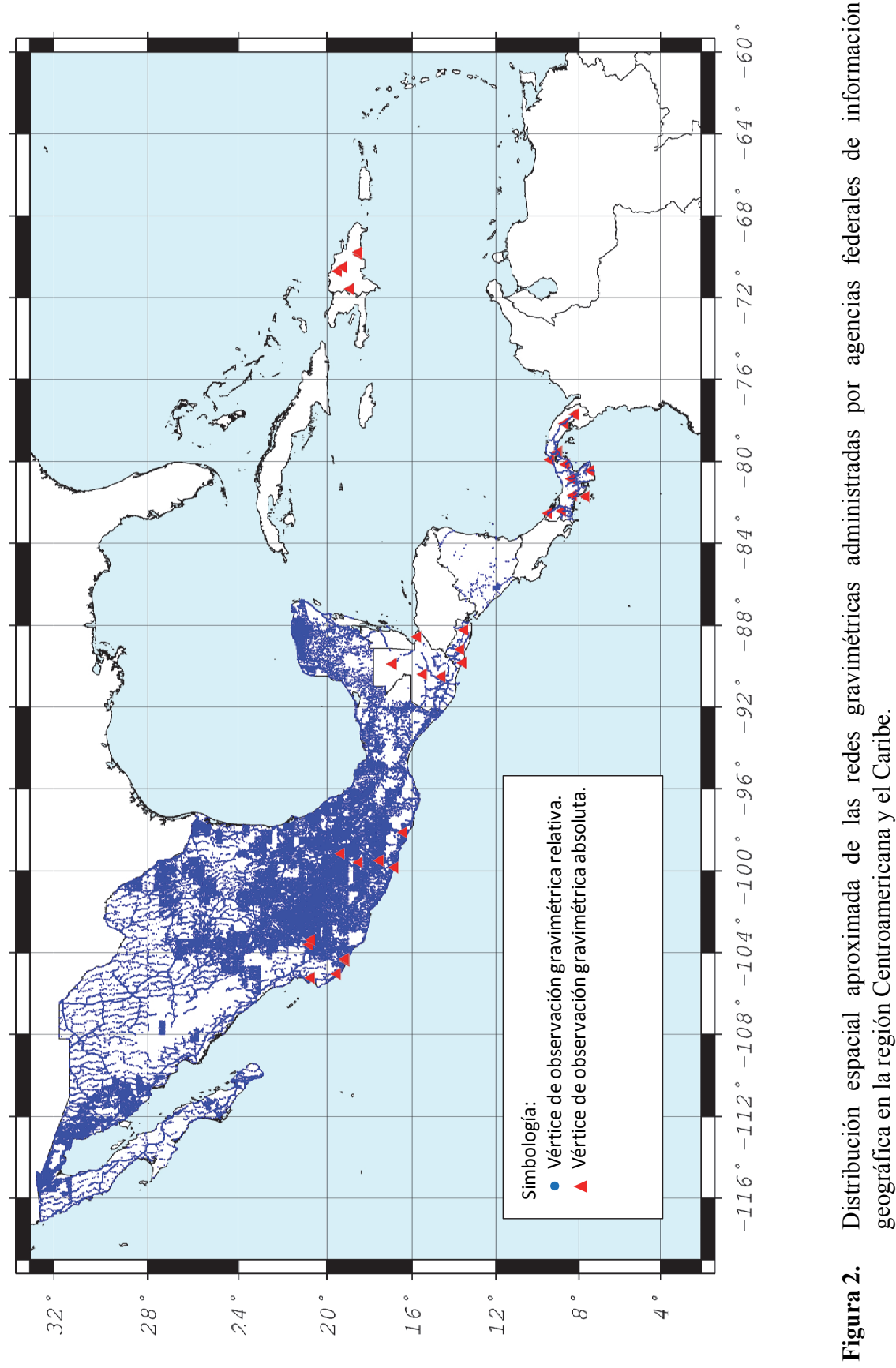




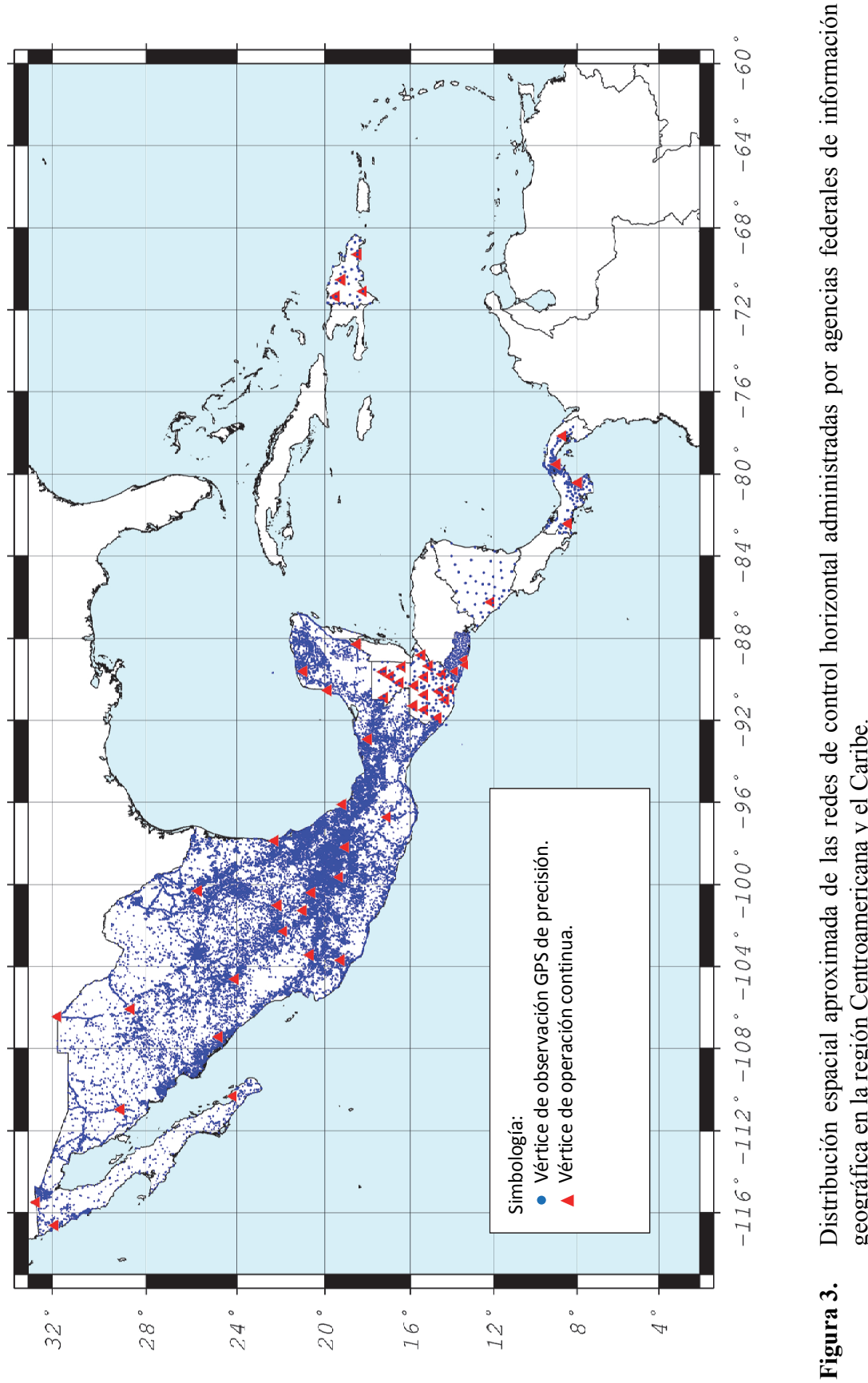




\section{Fuentes alternas}

La Tabla 2 indica casos en los que se tiene certeza de que organizaciones externas a las propias agencias sostienen actividades de gravimetría. Entre estas organizaciones se encuentran universidades, compañías petroleras y servicios privados de prospección geofísica. Dichas fuentes son reconocidas aunque se aclara que una cantidad mínima o nula de esos datos ha sido puesta a disposición de las agencias.

\section{Información específica por institución geográfica}

A continuación se presenta información particular por agencia que ayuda a identificar la situación y perspectiva de avances por cada una.

\section{Panamá}

La información gravimétrica en el IGN se administra en tres redes: red regional, red auxiliar y subase nacional. Estas se encuentran referidas a la Red Latinoamericana de Gravedad de 1977 (RELANG77), que a su vez está referida a puntos de la IGSN71. Los programas recientes de gravimetría han sido dirigidos a cubrir bancos de nivel. El nivel de precisión de las redes nacionales es actualmente indeterminado. Doce sitios de gravedad absoluta en el país fueron generados por el Instituto Nacional de Investigación Metrológica y el Centro Nacional de Metrología. Se espera avanzar en la recuperación y limpieza de los datos que servirán de base para tomar decisiones sobre futuros levantamientos.

\section{El Salvador}

En años recientes se han logrado avances concretos rumbo al establecimiento de un programa de levantamientos gravimétricos. En asociación con el National Geospatial Intelligence Agency de los Estados Unidos de América, el CNR mantiene actualmente un programa de levantamientos para la generación de una red densificada que apoyará tanto a la mejora de la red de control vertical como a futuros procesos de determinación del campo de gravedad. Se produjo la recuperación de los Bancos de Marca con datos de gravedad asociados que servirán de referencia para densificar en forma prioritaria la zona norte del país.

\section{México}

La información gravimétrica en el INEGI se administra en dos redes: red de estaciones base y red de densificación. Por norma éstas se encuentran referidas a la red IGSN71 y se estima que cuentan con un nivel de precisión de 0.02 y $0.05 \mathrm{mGal}$ respectivamente. Desde 1981 se ha mantenido en operación ininterrumpida el programa de densificación gravimétrica y se espera continuar incrementando su cubrimiento hasta lograr homogeneizar la densidad de mediciones en el territorio 
nacional. El avance actual en cubrimiento corresponde aproximadamente a 50\% del territorio nacional, adicionalmente se realizan levantamientos sobre bancos de nivel para facilitar la derivación de correcciones ortométricas. Se cuenta con información de ocho sitios observados con gravímetros absolutos electrónicos por parte del NGS en 1996. Se espera que estos sitios formen parte de la red fundamental y luego sirvan como referencia en estudios de exactitud regional de las redes principales.

\section{Nicaragua}

Los 52 vértices referidos en la Tabla 2 fueron establecidos con gravímetros relativos tomando como única referencia un vértice antiguo nombrado BM-C-B. Este vértice a su vez fue medido con un gravímetro absoluto de péndulo por parte del IAGS.

Los valores de aceleración de gravedad (g) asociados a cada sitio de observación de las redes gravimétricas provienen en su mayoría de levantamientos con gravímetros relativos. Las mediciones obtenidas de diferencias de gravedad $(\Delta \mathrm{g})$ incluyen generalmente correcciones tanto por factores de escala de los gravímetros como por el efecto de marea. La ecuación utilizada en la región para propagación de valores de gravedad se lee:

$$
g_{n}=g_{1}+\sum_{i-1}^{M} \Delta g_{i}
$$

la aceleración de gravedad de una marca numerada $n$ es igual al valor $g_{1}$ de la marca de partida que pertenece al marco de referencia nacional, más la suma de diferencias de gravedad $\Delta g_{i}$ medidas entre marcas consecutivas desde la primera hasta llegar a la marca $n$. Los valores $\Delta \mathrm{g}$ definitivos de cada levantamiento son generalmente derivados de un proceso de ajuste en gabinete.

\section{Tendencias regionales y globales}

Cabe apuntar que durante las últimas dos décadas los resultados de misiones satelitales con propósitos científicos han dejado un legado de mediciones cada vez más exacto sobre el campo de gravedad en cubrimiento global (ver por ejemplo las misiones CHAMPS, GRACE y GOCE). El estado del arte lo dictan ahora modelos geopotenciales globales como el llamado DIR3-GOCE, que hasta una resolución cercana a los $50 \mathrm{~km}$ logra representar fielmente el campo de gravedad.

Para fines de control geodésico ésta información tiene la ventaja de ser insesgada pero su relativamente baja resolución impide lograr exactitud en aplicaciones de alcance local. Añadiendo levantamientos de densificación por gravimetría terrestre puede lograrse una combinación que toma la exactitud global de los datos satelitales 
más la alta resolución de los levantamientos sobre la topografía. Para el caso de áreas con carencia de datos terrestres aún se aplican técnicas de interpolación por análisis de modelos digitales de elevación; sin embargo es bien conocido que al comparar los resultados contra modelos gravimétricos basados en densificación apropiada las faltas de exactitud son significativas.

De la información en la Tabla 2 puede notarse que la producción gravimétrica en gran parte de la región se encuentra detenida por insuficiencia presupuestal que inhibe la generación de proyectos. Esta es una situación que se procura revertir mediante apertura de comunicación interinstitucional que promueva la coordinación de esfuerzos. El Primer Taller Internacional de Monitoreo del Geoide para México, Centroamérica y el Caribe, que se llevó a cabo en septiembre de 2011 en Aguascalientes, México, representó el inicio del impulso a esta coordinación. Entre los logros obtenidos se cuenta la compartición de conocimientos en tratamiento de datos gravimétricos, el establecimiento de estándares estructurales para generar bases de datos, intercambio de reportes de avance y apoyo para producir metodologías de levantamientos (Avalos, 2011). Los resultados de este acercamiento demostraron la eficacia de esta estrategia. Actualmente esta comunicación continúa abierta con la ejecución de reuniones a distancia y nuevos talleres entre representantes de las agencias (Avalos, 2013).

\section{Discusión}

Grandes retos se detectaron al impulsar la comunicación interinstitucional. Cinco de las representaciones nacionales incluidas en este reporte cuentan con infraestructura gravimétrica en un estado de desarrollo frenado. Esto se atribuye primordialmente a una prolongada prevalencia de insuficiencia presupuestal, aunada con escacez de oportunidades para la capacitación. Este hecho se refleja en la información en las cuatro últimas columnas de la Tabla 2. No obstante, en referencias como la del párrafo anterior es notable que aún en esta situación la comunicación interinstitucional puede generar avances concretos. De ser alentado y expandido este tipo de colaboración, es probable que objetivos ambiciosos como la unificación regional de dátum gravimétrico y vertical sean alcanzados para evitar que la infraestructura geodésica instalada se convierta en un impedimento del desarrollo tecnológico en otros campos.

En cuanto al aspecto de monitoreo de cambios temporales en el campo de gravedad, es declarado que los programas de trabajo aún no contemplan este aspecto; sin embargo es recomendado que los proyectos futuros lo consideren. La región se caracteriza por alta actividad tectónica, lo que presupone la existencia de cambios significativos, capaces de afectar la precisión de los resultados en una red de precisión. Esfuerzos como el iniciado por el NGS en 1996, instalando vértices de grave- 
dad absoluta en varios países, son útiles sólo si se alienta su continuación para obtener re-observaciones cada cierto número de años. Otras técnicas de monitoreo basadas en observaciones relativas son susceptibles de análisis para su eventual implementación como parte complementaria de objetivos como la densificación gravimétrica (e.g. Pagiatakis, 2003).

\section{Infraestructura de referencia horizontal}

En la actualidad la tecnología GNSS representa la principal herramienta utilizada para construir marcos de referencia en la región. Dado que este hecho se encuentra ampliamente documentado por la acción de programas como SIRGAS (véase por ejemplo: Drewes et al., 2002; Seemuller et al. y 2008; Brunini et al., 2012), esta vertiente se aborda en forma breve. La atención aquí se centra en introducir la información de la Tabla 3, que ayuda a dar descripción general de la capacidad instalada y las perspectivas de crecimiento que se considera complementario a la documentación existente (véase Tabla 3 en "Tablas de resumen sobre infraestructura geodésica").

\section{Información específica por agencia}

A continuación información aclaratoria sobre los programas de trabajo y proyectos que inciden sobre las redes de referencia horizontal.

\section{Panamá}

En la actualidad existen cuatro CORS activas y se tienen 15 instaladas recientemente que se espera entren a fase de operación. Las redes horizontales están en un periodo de actualización de ITRF97 a ITRF08. Existe un proyecto de inversión para aumentar la cantidad de equipo receptor GNSS en soporte del mantenimiento de la red horizontal.

\section{Nicaragua}

Hasta 1983 se logró establecer un total de 677 vértices de referencia pasivos. En 1996 se crearon 52 estaciones adicionales. Actualmente se efectúan esfuerzos para identificar las marcas existentes.

\section{México}

La Red Geodésica Nacional Activa (RGNA) del INEGI mantiene en operación 26 estaciones fijas en México, aunque adicionalmente procesa información de ocho estaciones extranjeras para producir coordenadas de referencia IGS que son utiliza- 
das en el marco de referencia SIRGAS. El marco de referencia vigente está materializado por coordenadas obtenidas de procesamiento fiducial de cada estación. A partir de procedimientos estándar aprobados por el IGS se determinaron coordenadas basadas en soluciones diarias y en series de tiempo para proveer vectores de velocidad local. Simultáneamente el INEGI coordina esfuerzos con agencias estadounidenses y canadienses para el establecimiento del Marco de Referencia Norteamericano (NAREF). México cuenta también con un número indeterminado de estaciones operadas en iniciativa privada que son utilizadas para control geodésico de proyectos locales independientes.

\section{El Salvador}

Desde que se iniciaron las primeras mediciones de Redes Geodésicas Horizontales se logró establecer y mantener una red de aproximadamente 1,500 vértices geodésicos de primero y segundo orden en todo el país. Con la implementación de la tecnología GNSS en 1998 se diseñó una nueva red geodésica básica nacional de 16 puntos, la cual ha sido sustituida en el 2007 con una nueva red geodésica básica de 38 vértices que ha permitido realizar los cálculos para la implementación de un nuevo Sistema de Referencia Geocéntrico basado en SIRGAS y denominado SIRGAS_ES2007 al cual están vinculados un aproximado de 1,600 vértices geodésicos pasivos medidos en los últimos años y a los cuales se les da un mantenimiento constante. Se tiene previsto la instalación de tres o más estaciones de operación continua en poco tiempo.

\section{Discusión}

El uso de tecnología GNSS es claramente la alternativa estándar en la región como herramienta para el establecimiento de puntos de control; sin embargo los conceptos manejados para procesar la estructura de una red pueden diferir entre representaciones.

Analizando la información de la Tabla 3, puede decirse que la infraestructura administrada por las agencias acumula 43 estaciones de operación continua y cerca de 90,000 vértices de referencia pasivos. A pesar de que en la región se utilizan cuatro marcos de referencia distintos, solamente en Honduras y Nicaragua se da mantenimiento en forma oficial al marco NAD27 considerado incompatible con los demás, que son de dátum geocéntrico y con capacidad de dar soporte a aplicaciones con exactitud superior.

De la información sobre cantidad de equipos receptores y personal capacitado para operarlos cabe observar que en Guatemala y Honduras, por el gran territorio en su responsabilidad y su expansión urbana, la capacidad relativa es inferior, denotándose como áreas de oportunidad prioritarias. 


\section{Tablas de resumen sobre infraestructura geodésica}

A continuación se presentan tres tablas que sintetizan una cantidad considerable de información con respecto a la infraestructura geodésica existente en la región. Con motivo de apoyar al lector en la interpretación de estos datos, se enlistan las siguientes consideraciones:

a) Las cantidades plasmadas corresponden a infraestructura bajo la administración de las agencias. Es probable que las cifras varíen en corto plazo debido a que los programas de trabajo aumentan las bases de datos mientras que algunas marcas físicas en el terreno pueden llegar a ser destruidas.

b) Las columnas de información antigua/reciente hacen referencia a productos realizados con metodología, dispositivos o programas de trabajo fundamentalmente distintos. Esa separación advierte cambios relevantes que han marcado una época.

c) Los campos sin información se marcan con un símbolo "-", que significa ausencia de datos o no aplicabilidad del campo según los programas de trabajo existentes.

d) Las dos columnas finales muestran el tipo de requerimientos que puede producir una aceleración en el quehacer geodésico de cada agencia. Se sugiere que los objetivos de acuerdos interinstitucionales apunten a mitigar los obstáculos mencionados.

\section{Conclusiones}

Se ha presentado un recuento global de la infraestructura geodésica que a nivel federal se oferta como sustento al desarrollo dentro de la región de Centroamérica y el Caribe. Un total de ocho instituciones responsables del manejo de información geográfica nacional lograron concentrar su información para fines de valorar avances y facilitar la identificación de prioridades sobre el desarrollo esperado en corto y mediano plazos. Las representaciones que participaron corresponden a México, Guatemala, El Salvador, Honduras, Nicaragua, Costa Rica, Panamá y República Dominicana.

La infraestructura geodésica de control geodésico vertical en la región se compone de más de 34,000 bancos de nivel de primer orden, organizados en redes de cobertura nacional. Se presentaron también cifras sobre redes de segundo y tercer orden que apoyan a aplicaciones como la construcción de modelos digitales de elevación del terreno. Actualmente y en el corto plazo los programas de trabajo apuntan a reconstruir y/o densificar la red de líneas de nivelación. Se informó también la actual carencia de programas dirigidos a monitorear movimientos verticales de la corteza terrestre que influyen en la exactitud de las redes nacionales. 


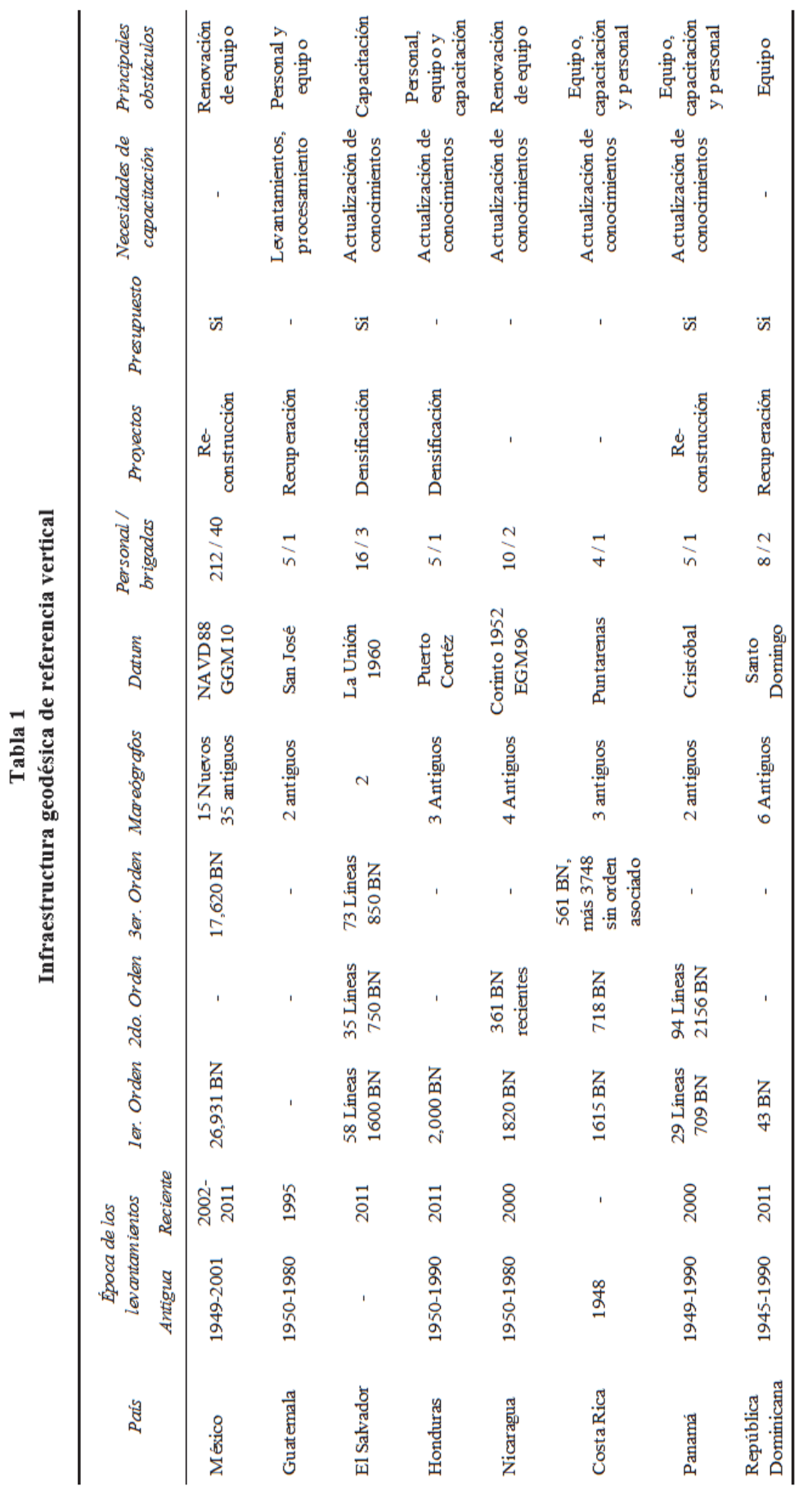




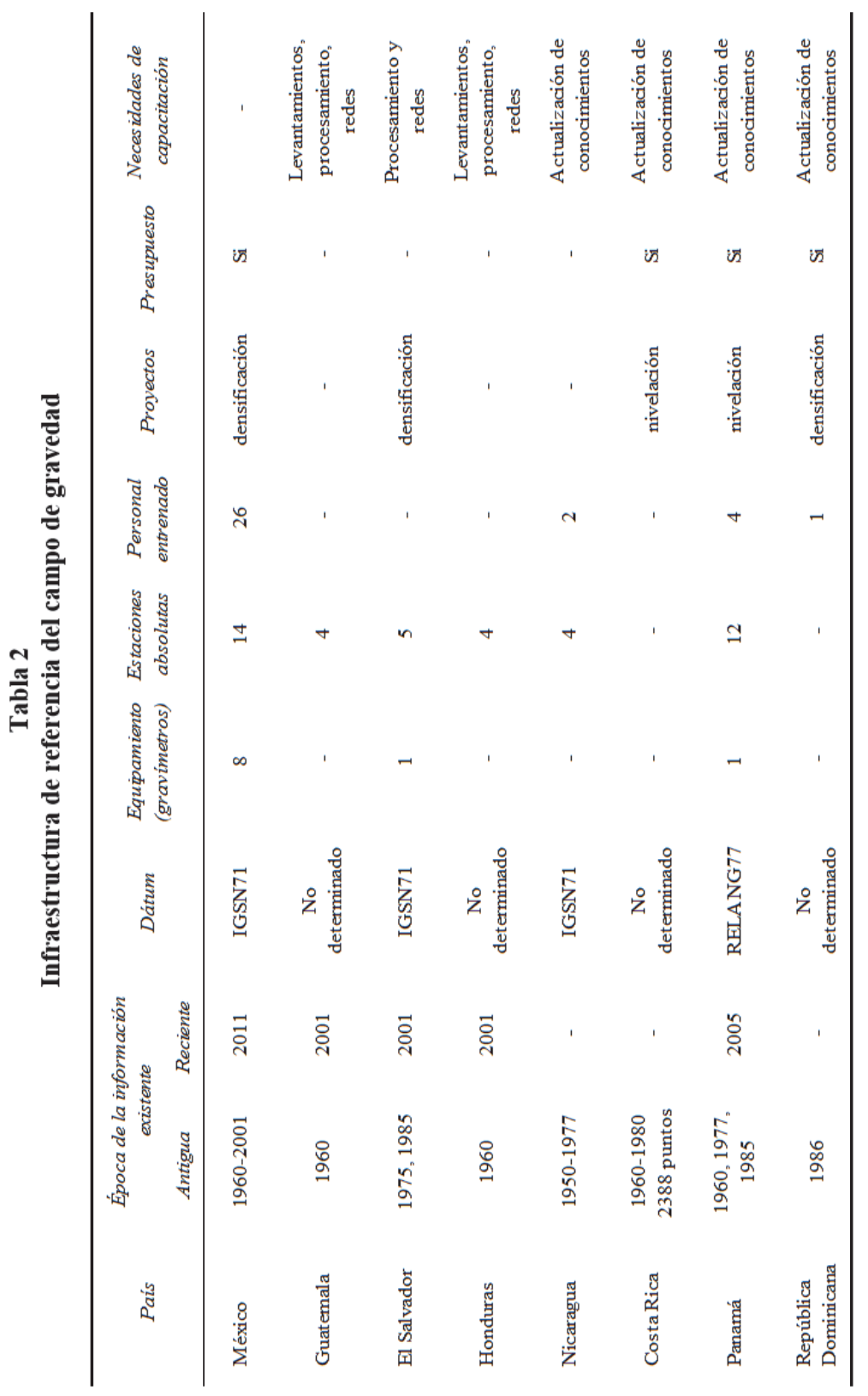




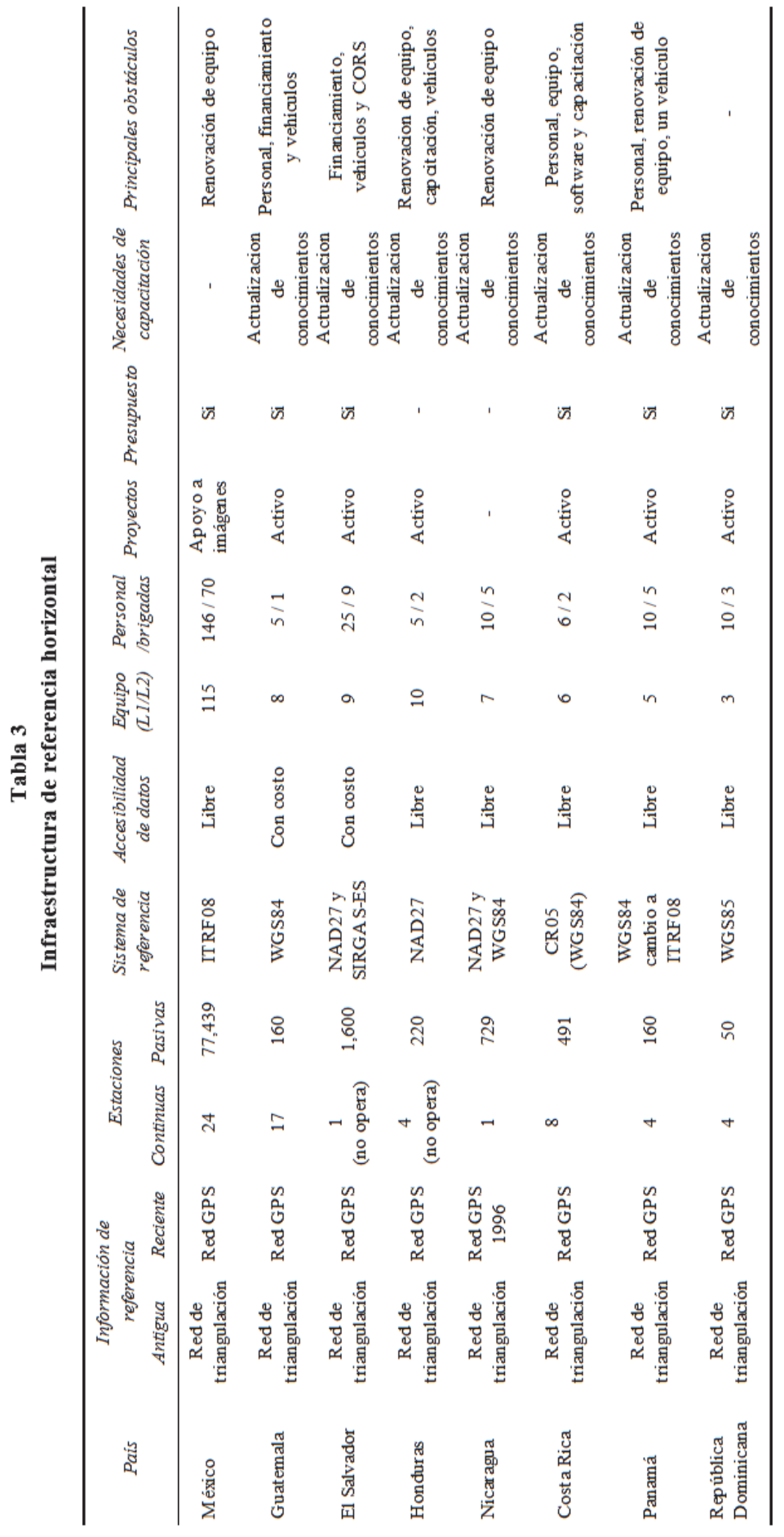


La infraestructura geodésica de control gravimétrico se compone de redes independientes entre países; aunque en los casos de México, El Salvador y Panamá existe una relación cercana por referirse a dátum compatibles como son IGSN71 y RELANG77. Tres de las ocho representaciones mantienen en operación programas de levantamientos que están dirigidos a reforzar el control geodésico vertical mediante procesos como cálculo de correcciones ortométricas y producción de modelos geoidales. Las cinco restantes reconocen la necesidad de impulsar su operación para mejorar el cubrimiento y modernizar el marco de referencia; sin embargo la insuficiencia presupuestaria frena la posibilidad de adquirir el equipo de medición.

La infraestructura geodésica de control horizontal se reportó con un total de 63 estaciones GNSS de operación continua en resguardo de las agencias representadas. La tecnología GNSS es un estándar en la región; sin embargo la metodología adoptada para el establecimiento de redes es diversa. Honduras y Nicaragua se listaron como las representaciones con mayores retos para ampliar la cobertura del servicio. Aun así queda asentado que la actividad de mantenimiento y expansión de las redes de control horizontal se encuentra mayormente soportada por programas y proyectos activos y continuos en contraste al soporte que se brinda a las actividades de gravimetría y control vertical.

En forma general consideramos que la capacidad instalada en la región ha crecido en un ritmo que logra satisfacer requerimientos mínimos de operación. Los datos presentados en forma tabular y las discusiones ofrecidas forman evidencia de que las necesidades de desarrollo en el futuro de mediano plazo permanecen sin garantía de ser cubiertas. Iniciativas como el proyecto SIRGAS y el proyecto del IPGH-INEGI, llamado Monitoreo del geoide para México, Centroamérica y el Caribe han incidido positivamente en la mejora de infraestructura y en organización de bases de datos. Estos esfuerzos han probado su efectividad en promover la adaptación de los institutos geográficos a la adopción de nuevas tendencias tecnológicas.

En cuanto a limitantes y prioridades detectadas, se documentó que la falta de capacitación y equipo renovados son frenos principales causados por insuficiencia presupuestal. Con el fin de mitigar esta situación se recomienda continuar incentivando la capacitación especializada, desde la cual el personal de las agencias podrá producir proyectos locales encaminados a equilibrar los avances en las tres vertientes del control geodésico.

\section{Bibliografía}

Amos, M., 2010. "New Zeland vertical datum 2009", New Zeland Surveyor, Journal of the New Zeland Institute of Surveyors, No. 300. 
Avalos, D., 2011. Informe técnico de actividades y resultados del proyecto Monitoreo del geoide para México, Centroamérica y el Caribe, Instituto Nacional de Estadística y Geografía, Aguascalientes, México, septiembre.

— 2013. "Segundo Taller del geoide para México, Centroamérica y el Caribe", Informe de actividades y resultados, Instituto Nacional de Estadística y Geografía, Aguascalientes, México, noviembre.

Baker, T. F., 1993. "Absolute sea level measurements, climate change and vertical crustal movements", Global and Planetary Change, No. 8, Elsevier, Amsterdam.

Brunini C., y Sánchez L., 2012. "Geodetic activities in Latin America and The Caribbean: always IN", Coordinates, Vol. VIII, Issue 6, 14-21, June.

Curch, J. A. and N. J. White, 2006. "A 20th century acceleration in global sea-level rise", Geophysical Research Letters, Vol. 33.

Drewes, H., L. Sánchez, D. Blitzkow y S. Freitas (2002). "Scientific foundations of the SIRGAS vertical reference system. Springer", IAG Symposia, Vol. 124:297301.

Foreman, M. G. G., W. R. Crawford, J. Y. Cherniawsky y J. Galbraith, 2008. "Dynamic ocean topography for the northeast Pacific and its continental margins", Geophysical Research Letters, Vol. 35.

Gibbons Media \& Research LLC, 2013. "How do GNSS-derived heights differ from other height systems", GNSS Solutions, issue May/June.

Hirt, C., M. Schmitz, U. Feldmann-Westendorff, G. Wübbena, C. H. Jahn y G. Seeber, 2011. "Mutual validation of GNSS height measurements and highprecision geometric-astronomical leveling", GPS Solutions, Vol. 15, No. 2, April.

IPCC, 2007. Cambio Climático 2007, Informe de síntesis. Contribución de los Grupos de trabajo I, II, y III al Cuarto Informe de evaluación del Grupo Intergubernamental de Expertos sobre el Cambio Climático, editado por R. K. Pachouri y A. Reisinger, Ginebra, Suiza.

Kingdon, R., P. Vanicek, M. Santos, A. Ellmann y R. Tenzer, 2005. "Toward an improved orthometric system for Canada", Revista Geomática, Canadian Institute of Geomatics, Vol. 59.

Pagiatakis, S., 2009. "Determination of sea surface topography from tide gauge and atmospheric data", Oral presentation at the American Geophysical Union spring meeting.

Peltier, W. R., 2001. "On eustatic sea level history: Last Glacial Maximum to Holocene", Quaternary Science Reviews.

Santos, M., P. Vanicek, W. E. Featherstone, R. Kingdon, A. Ellmann, B. A. Martin, M. Kuhn, R. Tenzer, 2006. "The relation between rigorous and Helmert's defi- 
nitions of orthometric heights", Journal of Geodesy, Vol. 80, No. 12, pp. 691704.

Seemüller, W., M. Krügel, L. Sánchez, 2008. "The position and velocity solution DGF08P01 of the IGS Regional Network Associate Analysis Centre for SIRGAS (IGS-RNAAC-SIR)", DGFI Report No. 79, Munich, 110 pp.

Woodworth, P. L., 2008. "Monitoring and predicting long term global sea and land level changes", Presentación del Permanent Service for Mean Sea Level (PSMSL), Reino Unido.

Wziontek, H., Wilmes, H., Bonvalot, S., 2009. "AGrav - An International Database for Absolute Gravity Measurements", Poster presented at the IAG 2009 Scientific Meeting, Buenos Aires, Aug. 31-Sept. 4. 